\title{
Sevoflurane promotes the proliferation of HUVECs by activating VEGF signaling
}

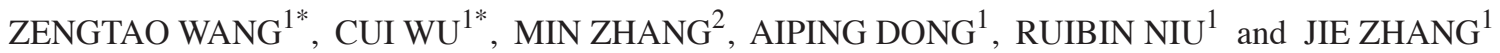 \\ ${ }^{1}$ Department of Anesthesiology, Huashan Hospital-North Fudan University; ${ }^{2}$ Department of Anesthesiology, \\ Central Hospital of Shanghai Yangpu District Affiliated to Tongji University, Shanghai 201907, P.R. China
}

Received April 9, 2018; Accepted November 5, 2019

DOI: $10.3892 /$ etm.2019.8319

\begin{abstract}
The vascular endothelium plays an essential role in vascular disease and cardiovascular diseases. The effects and underlying mechanisms of sevoflurane on vascular endothelial growth factor (VEGF) in human endothelial cells have not been elucidated. The MTT colorimetric assay was used to determine HUVEC activity at different concentrations (1 and 3\%, respectively) of sevoflurane for different time-points (12, 24 and $48 \mathrm{~h}$, respectively). The regulation of sevoflurane on the mRNA levels of VEGFa, VEGFb, VEGFc and VEGFR1,2,3 was analyzed by real-time PCR. When VEGFR2 was inhibited by axitinib, VEGFR2 protein expression was determined by western blotting, and the cell viability was assessed by MTT analysis. The results revealed that sevoflurane increased cell viability in a dose- and time-dependent manner. Sevoflurane significantly upregulated VEGFA mRNA expression only. In addition, sevoflurane increased the expression of VEGFR2 at the mRNA and protein levels, whereas sevoflurane did not modulate the mRNA expression of VEGFR1 and VEGFR3. Furthermore, sevoflurane failed to increase the mRNA and protein expression of VEGFR2 when VEGFR2 was inhibited by axitinib, an inhibitor of VEGF receptors. In conclusion, sevoflurane may be a promising agent against endothelium dysfunction-caused vascular disease by activating the VEGF-A/VEGFR2 signaling pathway.
\end{abstract}

\section{Introduction}

The endothelium presents a single-cell lining on the internal surface of blood vessels, cardiac valves, and numerous body cavities. The vascular endothelium has been considered as a multifunctional organ, which protects the vessel wall from

Correspondence to: Dr Jie Zhang, Department of Anesthesiology, Huashan Hospital-North Fudan University, 108 Luxiang Road, Baoshan, Shanghai 201907, P.R. China

E-mail: zhangjiew1@yeah.net

*Contributed equally

Key words: sevoflurane, vascular endothelial growth factor, VEGF receptor, VEGF signaling, HUVECs the vascular tone, vessel wall inflammation, and thrombosis resistance (1), and the endothelium participates in new vessel formation (2). Thus, the typical vascular function needs to keep the integrity of the vascular endothelium and a well-balanced release of numerous vasoactive substances (3). Endothelial dysfunction underlies the pathogenesis of vascular disease and cardiovascular diseases, such as coronary artery disease, coronary artery spasm, and atherosclerosis (3-5). Endothelial dysfunction has always been caused by reduced levels and adhesive function of circulating endothelial progenitor cells, which accelerates re-endothelialization $(6,7)$. Previous studies have revealed that angiogenesis is a physiological process involving the growth of new blood vessels either from endothelial cell precursors or from the pre-existing vasculature, and the processes are regulated by various angiogenic growth factors, such as vascular endothelial growth factor (VEGF) (8). By binding to 1 of 3 cognate receptor tyrosine kinases (VEGF receptor 1-3), VEGF has been regarded as the most vital cytokine in enhancing endothelial cell growth. The VEGF-mediated signaling pathway exhibits a vital role in maintaining the structure and function of the vascular endothelium by promoting endothelial cell proliferation $(9,10)$.

Sevoflurane is a general anesthetic, and it has been commonly used in the anesthesia of young children and infants (11). Sevoflurane has exhibited activity against oxidative stress, inflammation, and it has been revealed to protect organs against stress-caused injury (12-14). Sevoflurane pretreatment significantly inhibited TNF- $\alpha$-induced permeability and p38 MAPK activation in rat pulmonary microvascular endothelial cells by decreasing ICAM-1 levels (15). Sevoflurane appears to offer a more stable heart rate profile compared with either isoflurane or desflurane (16). Notably, sevoflurane increases HUVEC proliferation and adhesion, in addition to the incorporation of tubular structures into endothelial progenitor cells (17). However, the effects and underlying mechanisms of sevoflurane on VEGF in human endothelial cells have not been elucidated. In the present study, the effects and molecular mechanisms of sevoflurane on the proliferation of human umbilical vein endothelial cells (HUVECs) were investigated.

\section{Materials and methods}

Cell culture. Human umbilical vein endothelial cells (HUVECs) were purchased from Gibco; Thermo Fisher Scientific, Inc. 
(cat. no. C0155C). Cells were cultured in Medium 200 (cat. no. M200500) supplemented with LSGS (cat. no. S00310; both from Gibco; Thermo Fisher Scientific, Inc.) according to the manufacturer's instructions. HUVECs were digested with Trypsin/EDTA at the appropriate confluency ( 70-80\%). Cells were cultured in an incubator under normal conditions or with sevoflurane treatment (1 and 3\%). Treatment with sevoflurane was performed according to a previously reported method (18) and was achieved by connecting the incubator with the sevoflurane vaporizer (Abbott Laboratories) attached to the anesthetic machine (Dräger). The infrared gas analyzer (Puritan-Bennett) was used to monitor the sevoflurane concentration at the inflow and outflow connectors.

Cell viability assay. Cell viability was performed by MTT assay (cat. no. KA1606; Abnova). HUVECs were seeded in a 96-well plate at 2,000 cells/well under different conditions for 12, 24 48, and $72 \mathrm{~h}$. Reagent medium (15/80 $\mu \mathrm{l}$ per well) was added followed by incubation for $4 \mathrm{~h}$ at $37^{\circ} \mathrm{C}$. For the treatment with the VEGFA antibody, the cells were incubated with the antibody (20 $\mu \mathrm{M}$; cat. no. AF-493-NA; R\&D Systems) to chelate the effects of VEGFA in the culture medium and the corresponding control antibody $(20 \mu \mathrm{M}$; cat. no. AB-108-C; $\mathrm{R} \& \mathrm{D}$ Systems) was applied as a control during the exposure of sevoflurane. Then $100 \mu \mathrm{l}$ of the solubilizer was added to each well. OD570 $\mathrm{nm}$ was measured for each well on an absorbance plate reader. The cell viability was calculated by the ratio of OD570 at each time-point to OD570 at $0 \mathrm{~h}$ of each well and presented as the percentage of the ratio.

Quantitative RT-PCR. Total RNA was extracted from HUVECs using TRIzol reagent (Invitrogen; Thermo Fisher Scientific, Inc.). For mRNA analysis, $2 \mu \mathrm{g}$ total RNA was reverse-transcribed to cDNA using the PrimeScript ${ }^{\circledR}$ reagent kit (Takara Bio, Inc.). Quantitative PCR for mRNA was performed using SYBR-Green (Takara Bio, Inc.) with an ABI 7900 Real-Time PCR system (Applied Biosystems; Thermo Fisher Scientific, Inc.). The thermocycling conditions were as follows: Initial denaturation at $95^{\circ} \mathrm{C}$ for $3 \mathrm{~min}$; followed by 40 cycles of $95^{\circ} \mathrm{C}$ for $10 \mathrm{sec}, 60^{\circ} \mathrm{C}$ for $5 \mathrm{sec}$ and final extension at $72^{\circ} \mathrm{C}$ for $10 \mathrm{sec}$. Quantification of the mRNA expression was normalized to $\beta$-actin. The fold-change of the mRNA levels relative to the control cells was calculated using the $2^{-\Delta \Delta \mathrm{Cq}}$ method (19). The sequences of the human-specific primers were as follows: VEGFa forward, 5'-CGAAGAGAAGAGACACATTG-3' and reverse, 5'-GGA TGGAGGAAGGTCAAC-3'; VEGFb forward, 5'-ACAGGA CAGAGTTGGAAGA-3' and reverse, 5'-GGAAGAGCC AGTTGTAAGAT-3'; VEGFc forward, 5'-TGTGTCCAGTGT AGATGAAC-3' and reverse, 5'-TCTTCTGTCCTTGAGTTG AG-3'; VEGFR1 forward, 5'-ACTCGTGGCTACTCGTTA-3' and reverse, 5'-ACCTTGCTTCGGAATGATT-3'; VEGFR2 forward, 5'-ACTGTCATCCTTACCAATCC-3' and reverse, 5'-CCTCCAACTGCCAATACC-3' and VEGFR3 forward, 5'-ATGCGAATACCTGTCCTAC-3' and reverse: 5'-GTT GCCGATGTGAATGAG-3'.

siRNA transfection. Cells subjected to siRNA transfection were firstly cultured in half the volume of the culture medium and transfected with $3 \mathrm{ng} / \mathrm{ml}$ siRNA (sense, 5'-GCAGCGACAAGG

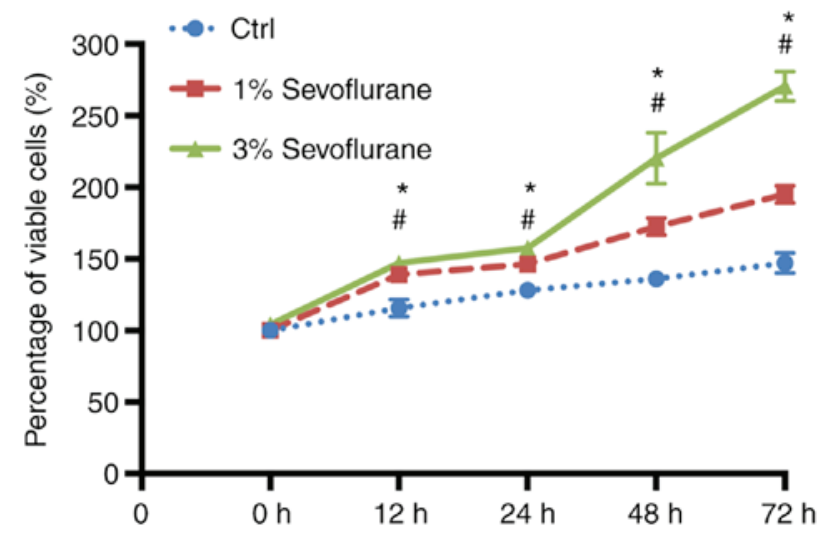

Figure 1. Sevoflurane increases the viability of HUVECs in a dose- and time-dependent manner. HUVECs were incubated in the absence or presence of 1 , and $3 \%$ concentrations of sevoflurane for various time-points and cell viability was determined by MTT assay. Values represent the means $\pm \mathrm{SD}$ of triplicate measurements. ${ }^{*} \mathrm{P}<0.05$ of $1 \%$ sevoflurane vs. the control group; ${ }^{\#} \mathrm{P}<0.05$ of $3 \%$ sevoflurane vs. the control group. Data represent three independent experiments. HUVEC, human umbilical cord endothelial cells.

CAGACUAUU-3' and antisense, 5'-UAGUCUGCCUUGUCG CUGCGU-3') or control siRNA (sense, 5'-UUCUCCGAACGU GUCACGUTT-3' and antisense, 5'-ACGUGACACGUUCGG AGAATT-3') (Shanghai GenePharma Co., Ltd.) by RNAiMAX (Invitrogen; Thermo Fisher Scientific, Inc.) for $8 \mathrm{~h}$ according to the product protocol. After $8 \mathrm{~h}$ of incubation, the other half of the culture medium was added, and cells were incubated for another $40 \mathrm{~h}$ so that the total interference lasted for $48 \mathrm{~h}$. After $48 \mathrm{~h}$ of incubation, the culture medium was changed to normal conditions without siRNA and subjected to further experiments.

Western blotting. Cells from each group were scraped in RIPA lysis buffer (Thermo Fisher Scientific, Inc.) with serine protease inhibitor. Proteins $(50 \mu \mathrm{g})$ were separated on $10 \%$ SDS-polyacrylamide gels and electroblotted onto polyvinylidene fluoride (PVDF) membranes (EMD Millipore). After incubating with blocking buffer, the membranes were incubated overnight at $4^{\circ} \mathrm{C}$ with the primary antibodies: Anti-VEGFR2 (dilution, 1:2,000; product code ab2349), anti-phosphorylated (p)-VEGFR2 (phosphorylated at Tyr 1175; dilution, 1:2,000; product code ab194806) and anti-beta Actin (dilution, 1:2,000; product code ab8226; all obtained from Abcam). Next, the membranes were washed and incubated with appropriate anti-rabbit HRP-conjugated secondary antibodies (dilution, 1:3,000; product code ab6728, Abcam) at $37^{\circ} \mathrm{C}$ for $30 \mathrm{~min}$. Protein bands were detected using the SuperSignal West Pico Chemiluminescent Substrate (Thermo Fisher Scientific, Inc.). The integrated optical density of the detected protein band was normalized to the control band for quantitative comparison by ImageJ (v. d1.47; National Institutes of Health).

VEGFR inhibitor. Axitinib, a VEGFR inhibitor, was purchased from Selleck Chemicals and was dissolved in DMSO at a stock concentration of $10 \mathrm{mM}$. The inhibitor was diluted to an appropriate final concentration in the culture medium.

Statistical analysis. All experiments were performed at least three times with data expressed as the mean \pm SEM or as otherwise stated. Statistical analyses were performed with 

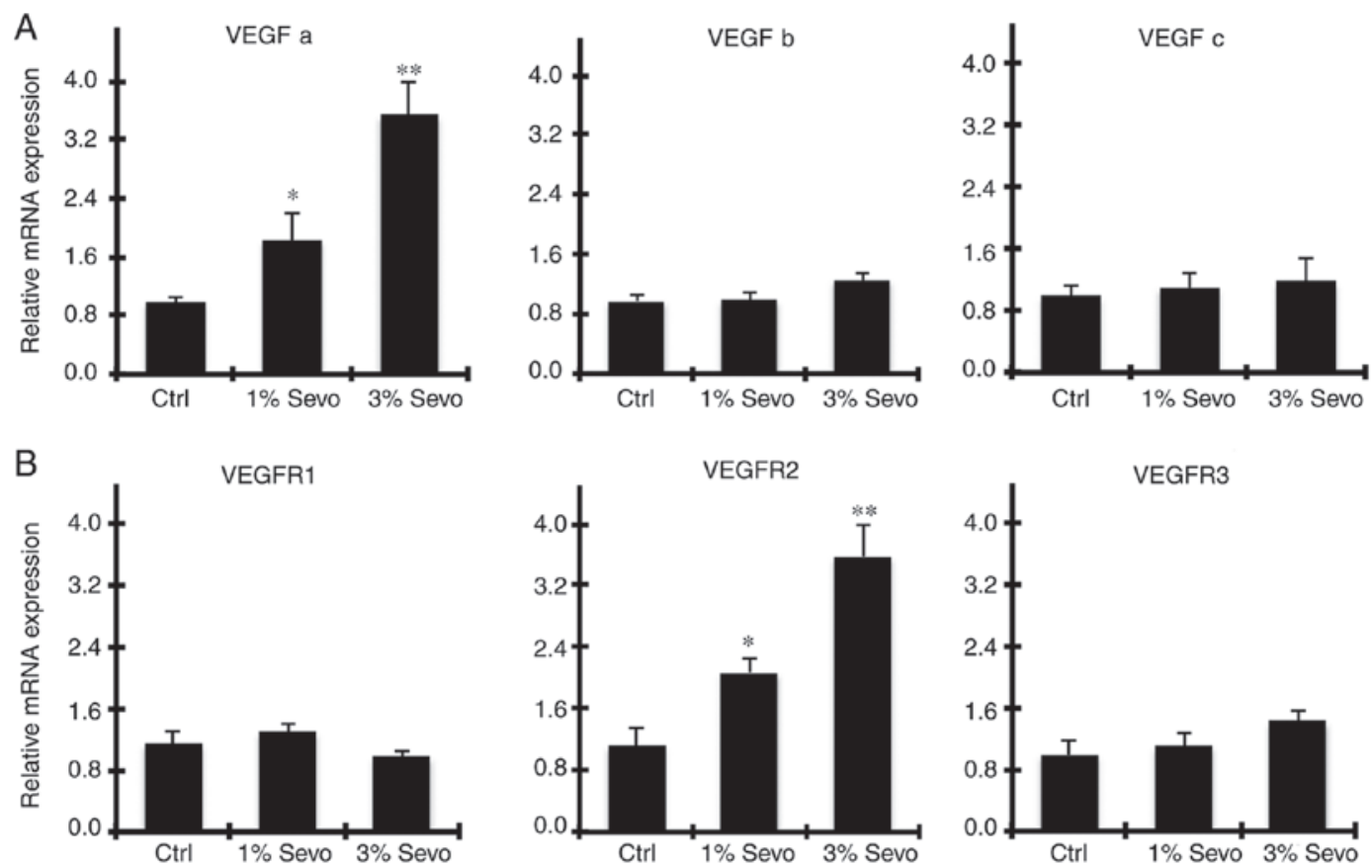

Figure 2. Sevoflurane induces the expression of VEGFa and VEGFR2. Reverse transcription-quantitative PCR analysis of (A) VEGFa, b, c and (B) VEGFR1, 2, 3 expression in HUVECs exposed to the absence or presence of 1 and 3\% concentrations of sevoflurane. Only VEGFa and VEGFR2 levels were induced by sevoflurane. Data represent the means $\pm \mathrm{SD}\left(\mathrm{n}=3\right.$ for each group, $\left.{ }^{*} \mathrm{P}<0.05,{ }^{* *} \mathrm{P}<0.01\right)$. Data represent three independent experiments. VEGF, vascular endothelial growth factor; VEGFR, VEGF receptor; HUVEC, human umbilical cord endothelial cells.

GraphPad Prism software (version 7; GraphPad Software, Inc.). Group comparisons were performed using one-way ANOVA with post hoc test using Dunnett's test or Sidak's multiple comparisons test. The two-column comparison was performed using Student's t-test. $\mathrm{P}<0.05$ or $\mathrm{P}<0.01$ were considered to indicate a statistically significant difference ( $\mathrm{P}<0.05$ and ${ }^{* *} \mathrm{P}<0.01$, as indicated in the figures and legends).

\section{Results}

Sevoflurane promotes the proliferation of human umbilical vein endothelial cells (HUVECs). To investigate whether sevoflurane was able to stimulate proliferation of HUVECs, the HUVECs were cultured in different concentrations of sevoflurane. The MTT colorimetric assay was used to determine HUVEC activity at different concentrations (1 and 3\%, respectively) of sevoflurane at different time-points (12, 24, 48 and $72 \mathrm{~h}$, respectively). The results revealed that sevoflurane increased cell viability in a dose- and time-dependent manner (Fig. 1).

Sevoflurane increases VEGF signaling systems in HUVECs. The VEGF pathway has been demonstrated to serve essential roles in promoting HUVEC proliferation (5). The most well-known VEGFs and their receptors (VEGFRs) include VEGFa, b, and VEGFR-1, 2, 3. Thus qPCR was used to examine the expression levels of VEGFs and VEGFRs of HUVECs treated with 1, and 3\% sevoflurane, respectively. As revealed in Fig. 2A, compared with the control group, only the expression level of VEGFa was increased after exposure to sevoflurane for $48 \mathrm{~h}$, and the increase was in a concentration-dependent manner ( $\mathrm{P}<0.05$; Fig. 2A). Similarly, VEGFR2 expression was significantly increased after sevoflurane exposure $(\mathrm{P}<0.05$; Fig. $2 \mathrm{~B})$. These data indicated that sevoflurane induced the expression of VEGFa, which may regulate VEGFR2 and activation of the following signaling pathway.

$V E G F \alpha$ inhibition protects the proliferation of HUVECs exposed to sevoflurane. To explore whether VEGFa secreted by HUVECs is involved in regulating the cell proliferation under sevoflurane exposure, HUVECs in the presence of 1 and $3 \%$ sevoflurane were transfected with VEGFa siRNA. The interference efficiency is presented in Fig. S1. The data revealed that silenced expression of VEGFa resulted in decreased Ki67-positive HUVECs (Fig. 3A and B). Moreover, the decreased expression of VEGFa exhibited a similar effect on the viability of cells (Fig. 3C and D). To further assess whether VEGFa was sufficient to target sevoflurane stimuli, a VEGFa chelation experiment was performed in cell culture using anti-VEGFa antibodies. As revealed in Fig. $3 \mathrm{E}$ and $\mathrm{F}$ the proliferation of HUVECs under either 1 or $3 \%$ sevoflurane medium was significantly blocked by the addition of anti-VEGFa while the group receiving control IgG antibodies exhibited high proliferation. These data indicated that the enhanced proliferation of HUVECs due to the effect of sevoflurane was regulated by the expression level VEGFa.

Sevoflurane promotes the proliferation of HUVECs by activating the VEGF/VEGFR signaling pathway. To investigate whether VEGF signaling was the underlying mechanism of sevoflurane in enhancing HUVEC proliferation, a small molecular VEGFR inhibitor, axitinib, was used to block the VEGFR signaling pathway. Firstly, western blotting was used to assess the levels of VEGFR and p-VEGFR after exposure of HUVECs to different concentrations of Sevoflurane with VEGFR inhibitor for $48 \mathrm{~h}$. As revealed in Fig. 4A and B, the 
A

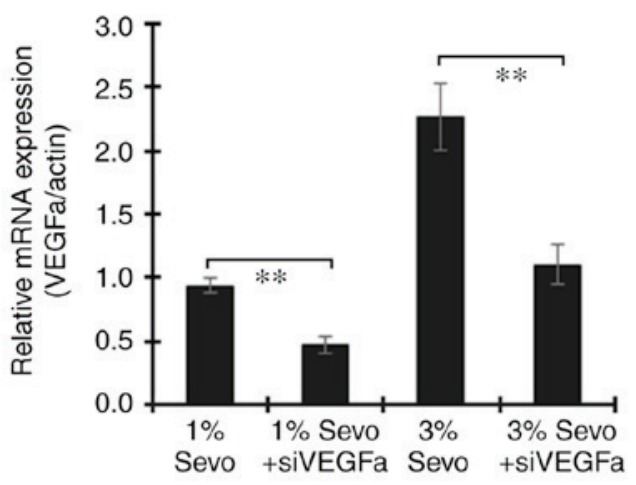

C

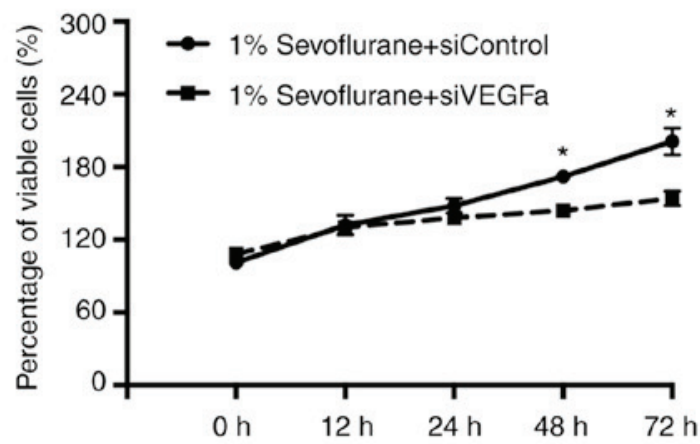

E

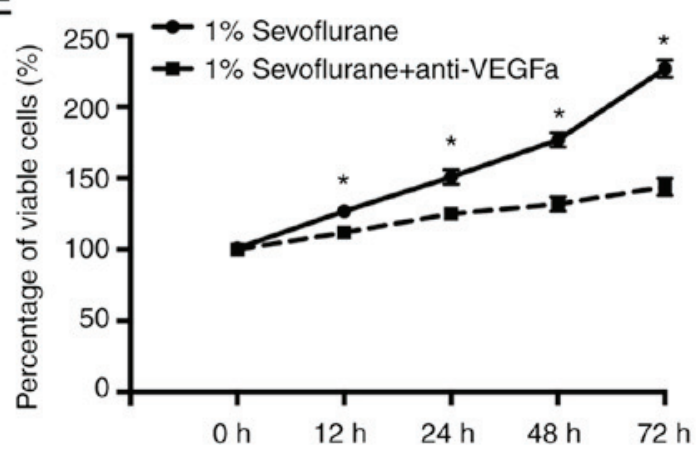

B
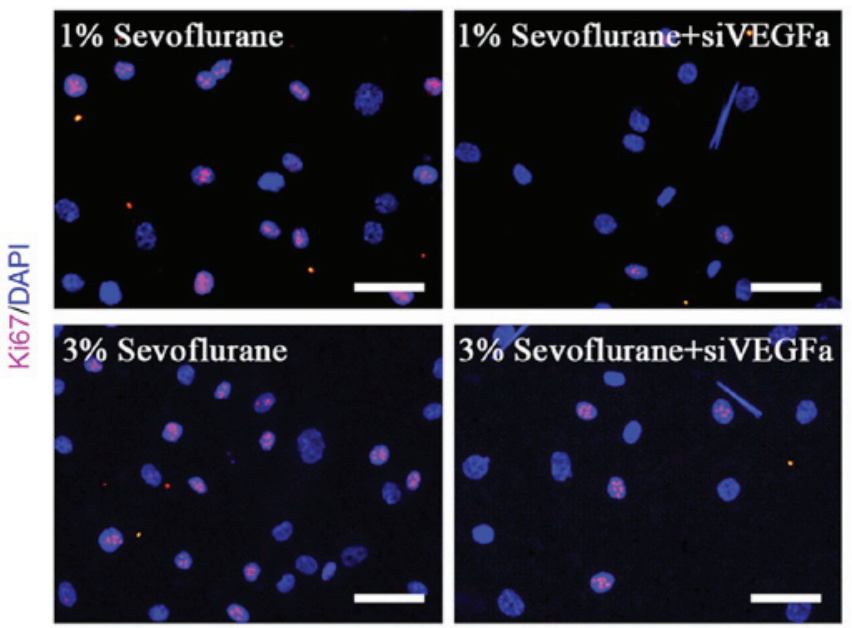

D

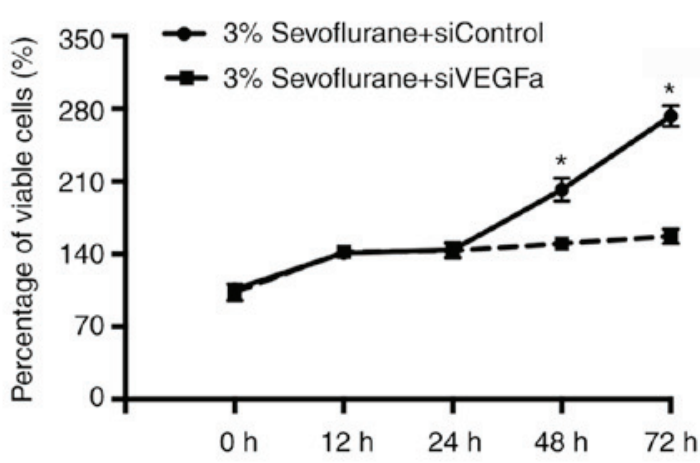

F

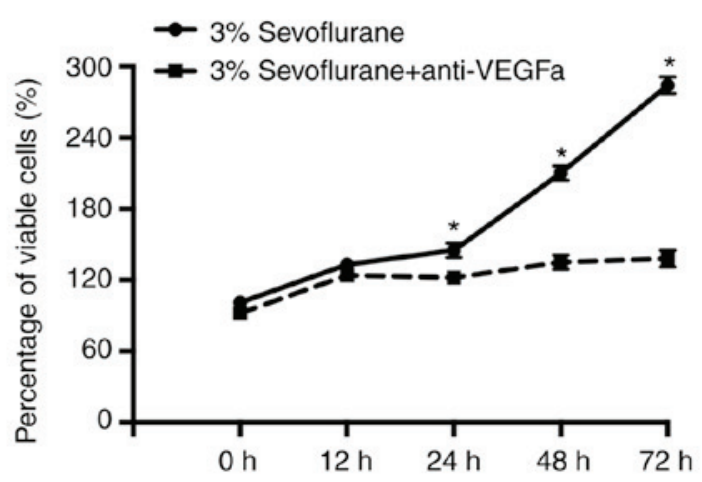

Figure 3. VEGFa inhibition protects the proliferation of HUVECs exposed to sevoflurane. HUVECs in the presence of 1 and 3\% sevoflurane were transfected with VEGFa siRNA. (A and B) The data revealed that abrogated expression of VEGFa could result in the decreased Ki67-positive HUVECs. (C and D) Depletion of VEGFa revealed a similar effect on proliferation. (E and F) The increased proliferation of HUVECs incubated in the presence of 1 and 3\% sevoflurane was chelated by anti-VEGFa at $0.1 \mu \mathrm{g} / \mathrm{ml}$ concentrations. Data represent three independent experiments. VEGF, vascular endothelial growth factor; HUVEC, human umbilical cord endothelial cells; siRNA, small interfering RNA.

significant decrease of the p-VEGFR level was observed in HUVECs exposed to axitinib and sevoflurane when compared with cells treated with sevoflurane alone. Notably, following the inhibition of the VEGFR pathway, there was significantly decreased cell proliferation in the cells treated with axitinib plus sevoflurane than with sevoflurane alone (Fig. 4C and D), implying that sevoflurane promotes the proliferation of HUVECs via activation of the VEGF/VEGFR pathway.

\section{Discussion}

The endothelium is involved in new vessel formation (2). The integrity of the vascular endothelium and an appropriate release of numerous vasoactive factors contribute to normal vascular physiology, and its dysfunction may incur the pathogenesis of vascular disease and cardiovascular diseases, such as coronary artery disease, coronary artery spasm, and atherosclerosis $(3-5,20,21)$. One of the major therapeutic strategies for cardiovascular diseases is to maintain vessel function and promote vessel regeneration $(4,5,20)$. In the present study, it was revealed that sevoflurane significantly increased cell viability and proliferation of HUVECs in a dose- and time-dependent manner, indicating that sevoflurane may increase new vessel formation resulting in attenuation of vascular disease.

The establishment and remodeling of the vascular system are regulated by several secreted signaling molecules, including 
A

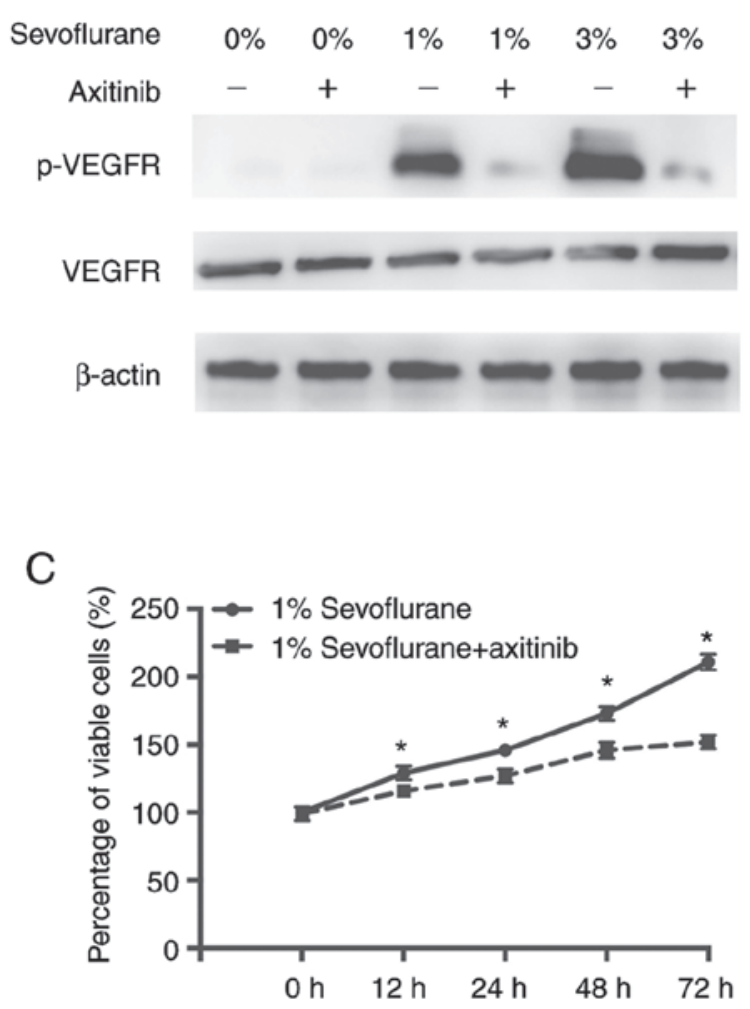

B

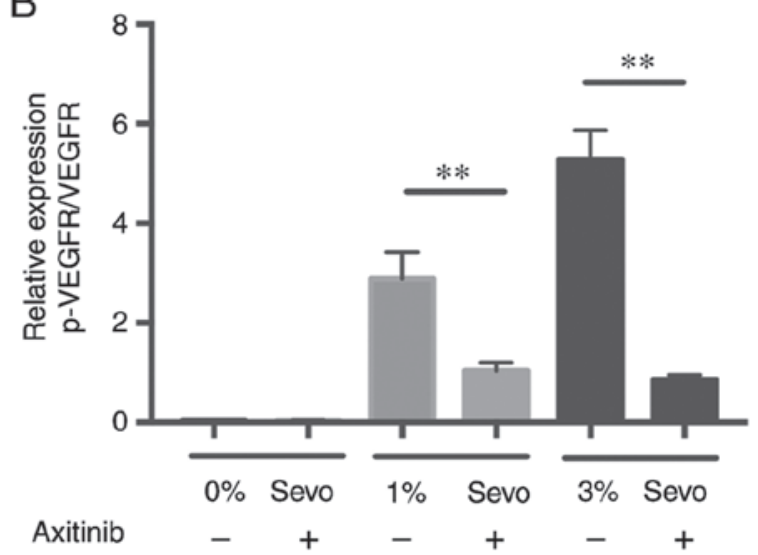

$\mathrm{D}$

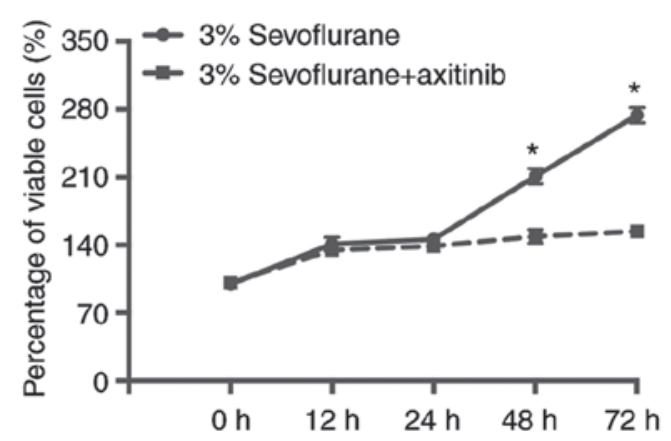

Figure 4. VEGF signaling is activated to promote the proliferation of HUVECs exposed to sevoflurane. (A) Representative western blots of VEGFR and p-VEGFR expression after exposure of HUVECs to 1 and $3 \%$ sevoflurane with and without the VEGFR inhibitor, axitinib. (B) The graph revealed the relative levels of phosphorylated VEGFR (p-VEGFR) normalized to VEGFR. Values without the inhibitor treatment were set as the baseline and considered equal to 1. (C and D) The MTT assay was used to detect cell viability of HUVECs incubated in (C) $1 \%$ and (D) $3 \%$ sevoflurane with and without the VEGFR inhibitor. Data are presented as the mean $\pm \mathrm{SD}$. ${ }^{*} \mathrm{P}<0.05$ vs. $1 \%$ sevoflurane and ${ }^{* *} \mathrm{P}<0.01$. Data represent three independent experiments. VEGF, vascular endothelial growth factor; VEGFR, VEGF receptor; HUVEC, human umbilical cord endothelial cells.

VEGF, and their corresponding receptors. They mediate downstream signaling that can result in the regulation of endothelial cell number and function $(8,22-24)$. The VEGF gene undergoes alternative splicing to form 6 isoforms, VEGFa, VEGFb, VEGFc, VEGFd, VEGFe, and VEGFf, where VEGFa is the most biologically active among them (25). VEGF receptors are classified into three groups, VEGFR1, VEGFR2, and VEGFR3 (26,27). VEGF-A and VEGF-B always bind to VEGFR1, VEGFa and VEGFe frequently bind to VEGFR2, whereas VEGFc and VEGFd mainly bind to VEGFR3 (28). Previous studies have revealed that VEGFR1 [also known as Fms-related tyrosine kinase 1 (FLT1)] plays an essential role in vessel formation $(10,29,30)$. Depletion of VEGFR1 has been revealed to cause early embryonic lethality at embryonic day 8.5 to 9 by enhancing endothelial cell overgrowth and excessive VEGFR2 [also known as kinase insert domain receptor, (KDR)] activation, which results in increased hemangioblast commitment and disorganized vessel formation $(10,29,30)$. This suggests that VEGFR1 negatively regulates VEGFR2 signaling by sequestering VEGF and preventing it from binding to VEGFR2, which is the primary receptor to modulating angiogenesis by elevating the cell proliferation/survival, migration, and differentiation of the endothelium (10,29-31). VEGFR2-null mice die at embryonic day 8.5 to 9 by increasing the defective blood-island formation and nearly absence of vasculature $(32,33)$. In addition, VEGFR3 (FLT-4), which may be activated by VEGF-C, has been revealed to play a vital role in establishing and maintaining lymphatic endothelial cells (34), and VEGFR3-mediated endothelial Snail was revealed to regulate capillary branching morphogenesis in the retina $(35,36)$. Furthermore, clinical studies demonstrated that VEGF inhibition was associated with cardiovascular disease $(37,38)$. It has been reported that sevoflurane exhibits its protective effects on pulmonary microvascular endothelial cells by downregulating ICAM-1 expression and inhibiting TNF- $\alpha$ and p38 MAPK signaling (15). In the present study, it was revealed that VEGF signaling could be activated by sevoflurane at a dose- and time-dependent manner in HUVEC cells. This was consistent with a previous study in which sevoflurane increased proliferation, adhesion on HUVECs, and incorporation in tubular structures of endothelial progenitor cells (17). Moreover, sevoflurane significantly increased the mRNA expression of VEGFa, however, sevoflurane did not affect VEGFb and VEGFc mRNA expression. In addition, sevoflurane upregulated the expression of VEGFR2 at the mRNA and protein levels, however it did not change the mRNA expression of VEGFR1 and VEGFR3. Furthermore, sevoflurane failed to elevate the VEGFR2 mRNA and protein expression when VEGFR2 was inhibited by axitinib, an inhibitor of VEGF receptors. These data indicated that sevoflurane could promote the proliferation of HUVEC cells by activating the VEGFa/VEGFR2 signaling pathway.

In conclusion, sevoflurane may be a promising agent against endothelium dysfunction-induced vascular disease by activating the VEGFa/VEGFR2 signaling pathway. 


\section{Acknowledgements}

Not applicable.

\section{Funding}

No funding was received.

\section{Availability of data and materials}

The datasets used and/or analyzed during the current study are available from the corresponding author on reasonable request.

\section{Authors' contributions}

ZW and $\mathrm{CW}$ performed majority of the experiments and contributed to the manuscript preparation. MZ and AD assisted with the animal experiments. RN performed the statistical analysis. JZ supervised the experiments and reviewed the manuscript. All authors read and approved the final manuscript.

\section{Ethics approval and consent to participate}

Not applicable.

\section{Patient consent for publication}

Not applicable.

\section{Competing interests}

The authors declare that they have no competing interests.

\section{References}

1. Davignon $\mathrm{J}$ and Ganz P: Role of endothelial dysfunction in atherosclerosis. Circulation 109: 27-32, 2004.

2. Yoder MC: Human endothelial progenitor cells. Cold Spring Harbor Perspect Med 2: a006692, 2012.

3. Chhabra N: Endothelial dysfunction-A predictor of atherosclerosis. Inter J Med Update 4: 33-41, 2009.

4. Park KH and Park WJ: Endothelial dysfunction: Clinical implications in cardiovascular disease and therapeutic approaches. J Korean Med Sci 30: 1213-1225, 2015.

5. Rajendran P, Rengarajan T, Thangavel J, Nishigaki $Y$, Sakthisekaran D, Sethi G and Nishigaki I: The vascular endothelium and human diseases. Int J Biol Sci 9: 1057-1069, 2013.

6. Werner N, Junk S, Laufs U, Link A, Walenta K, Bohm M and Nickenig G: Intravenous transfusion of endothelial progenitor cells reduces neointima formation after vascular injury. Circ Res 93: e17-e24, 2003.

7. Huang PH, Chen YH, Chen YL, Wu TC, Chen JW and Lin SJ: Vascular endothelial function and circulating endothelial progenitor cells in patients with cardiac syndrome X. Heart 93: 1064-1070, 2007.

8. Lin JM, Zhao JY, Zhuang QC, Hong ZF and Peng J: Xiongshao capsule promotes angiogenesis of HUVEC via enhancing cell proliferation and up-regulating the expression of bFGF and VEGF. Chin J Integr Med 17: 840-846, 2011.

9. Carratelli CR, Paolillo R and Rizzo A: Chlamydia pneumoniae stimulates the proliferation of HUVEC through the induction of VEGF by THP-1. Int Immunopharmacol 7: 287-294, 2007.

10. Hassel D, Cheng P, White MP, Ivey KN, Kroll J, Augustin HG, Katus HA, Stainier DY and Srivastava D: MicroRNA-10 regulates the angiogenic behavior of zebrafish and human endothelial cells by promoting vascular endothelial growth factor signaling. Circ Res 111: 1421-1433, 2012
11. Liu X, Song X, Yuan T, He J, Wang X and Wang Q: Effects of calpain on sevoflurane-induced aged rats hippocampal neuronal apoptosis. Aging Clin Exp Res 28: 633-639, 2016.

12. Hofstetter C, Boost KA, Flondor M, Basagan-Mogol E, Betz C, Homann M, Muhl H, Pfeilschifter J and Zwissler B: Anti-inflammatory effects of sevoflurane and mild hypothermia in endotoxemic rats. Acta Anaesthesiol Scand 51: 893-899, 2007.

13. Herrmann IK, Castellon M, Schwartz DE, Hasler M, Urner M, Hu G, Minshall RD and Beck-Schimmer B: Volatile anesthetics improve survival after cecal ligation and puncture. Anesthesiology 119: 901-906, 2013.

14. JulierK, da Silva R, GarciaC,BestmannL,FrascaroloP,Zollinger A, Chassot PG, Schmid ER, Turina MI, von Segesser LK, et al: Preconditioning by sevoflurane decreases biochemical markers for myocardial and renal dysfunction in coronary artery bypass graft surgery: A double-blinded, placebo-controlled, multicenter study. Anesthesiology 98: 1315-1327, 2003.

15. Sun SX, Ge BX and Miao CH: Effects of preconditioning with sevoflurane on TNF- $\alpha$-induced permeability and activation of p38 MAPK in rat pulmonary microvascular endothelial cells. Cell Biochem Biophys 61: 123-129, 2011.

16. Ebert TJ, Harkin CP and Muzi M: Cardiovascular responses to sevoflurane: A review. Anesth Analg 81 (Suppl 6): S11-S22, 1995.

17. Vlad AM, Isvoranu G, Gilca M, Ceafalan L, Surcel M, Stoian I and Manda G: Sevoflurane increases proliferation, adhesion on HUVEC and incorporation in tubular structures of endothelial progenitor cells. FASEB J 29: LB590, 2015.

18. Yang Y,Hu R, Yan J, Chen Z, Lu Y, Jiang J and Jiang H: Sevoflurane inhibits the malignant potential of head and neck squamous cell carcinoma via activating the hypoxiainducible factor-1 $\alpha$ signaling pathway in vitro. Int J Mol Med 41: 995-1002, 2018.

19. Livak KJ and Schmittgen TD: Analysis of relative gene expression data using real-time quantitative PCR and the 2(-Delta Delta C(T)) method. Methods 25: 402-408, 2001.

20. Saffi MA, Furtado MV, Polanczyk CA, Montenegro MM, Ribeiro IW, Kampits C, Haas AN, Rösing CK and Rabelo-Silva ER: Relationship between vascular endothelium and periodontal disease in atherosclerotic lesions: Review article. World J Cardiol 7: 26-30, 2015.

21. Meguro K, Iidaka T, Nakata M, Yamashita T, Chinen T, Fujita M, Kikuchi T, Keida T and Ohira H: Regular exercise habits and vascular endothelium function in patients with cardiovascular diseases. Eur Heart J 35: 730-731, 2014

22. Gerber HP, Vu TH, Ryan AM, Kowalski J, Werb Z and Ferrara N: VEGF couples hypertrophic cartilage remodeling, ossification and angiogenesis during endochondral bone formation. Nat Med 5: 623-628, 1999.

23. Dzietko M, Derugin N, Wendland MF, Vexler ZS and Ferriero DM: Delayed VEGF treatment enhances angiogenesis and recovery after neonatal focal rodent stroke. Transl Stroke Res 4: 189-200, 2013.

24. Lopes RA, Neves KB, Tostes RC, Montezano AC and Touyz RM: Downregulation of nuclear factor Erythroid 2-related factor and associated antioxidant genes contributes to redox-sensitive vascular dysfunction in hypertension. Hypertension 66: 1240-1250, 2015.

25. Neves KB, Rios FJ, van der Mey L, Alves-Lopes R, Cameron AC, Volpe M, Montezano AC, Savoia C and Touyz RM: VEGFR (Vascular Endothelial Growth Factor Receptor) inhibition induces cardiovascular damage via redox-sensitive processes. Hypertension 71: 638-647, 2018.

26. Ferrara N, Carver-Moore K, Chen H, Dowd M, Lu L, O'Shea KS, Powell-Braxton L, Hillan KJ and Moore MW: Heterozygous embryonic lethality induced by targeted inactivation of the VEGF gene. Nature 380: 439-442, 1996.

27. Carmeliet P, Ferreira V, Breier G, Pollefeyt S, Kieckens L, Gertsenstein M, Fahrig M, Vandenhoeck A, Harpal K, Eberhardt C, et al: Abnormal blood vessel development and lethality in embryos lacking a single VEGF allele. Nature 380: 435-439, 1996

28. Takahashi $\mathrm{H}$ and Shibuya M: The vascular endothelial growth factor (VEGF)/VEGF receptor system and its role under physiological and pathological conditions. Clin Sci (Lond) 109: 227-241, 2005.

29. Fong GH, Rossant J, Gertsenstein M and Breitman ML: Role of the Flt-1 receptor tyrosine kinase in regulating the assembly of vascular endothelium. Nature 376: 66-70, 1995.

30. Roberts DM, Kearney JB, Johnson JH, Rosenberg MP, Kumar R and Bautch VL: The vascular endothelial growth factor (VEGF) receptor Flt-1 (VEGFR-1) modulates Flk-1 (VEGFR-2) signaling during blood vessel formation. Am J Pathol 164: 1531-1535, 2004. 
31. Jiang JX, Chen XL, Serizawa N, Szyndralewiez C, Page P, Schröder K, Brandes RP, Devaraj S and Török NJ: Liver fibrosis and hepatocyte apoptosis are attenuated by GKT137831, a novel NOX4/NOX1 inhibitor in vivo. Free Radic Biol Med 53: 289-296, 2012.

32. Shalaby F, Rossant J, Yamaguchi TP, Gertsenstein M, Wu XF Breitman ML and Schuh AC: Failure of blood-island formation and vasculogenesis in Flk-1-deficient mice. Nature 376: 62-66, 1995.

33. Ranayhossaini DJ, Rodriguez AI, Sahoo S, Chen BB, Mallampalli RK, Kelley EE, Csanyi G, Gladwin MT, Romero G and Pagano PJ: Selective Recapitulation of conserved and Nonconserved regions of putative NOXA1 protein activation domain confers isoform-specific inhibition of Nox1 oxidase and attenuation of endothelial cell migration. J Biol Chem 288: 36437-36450, 2013.

34. Makinen T, Jussila L, Veikkola T, Karpanen T, Kettunen MI, Pulkkanen KJ, Kauppinen R, Jackson DG, Kubo H, Nishikawa $\mathrm{S}$, et al: Inhibition of lymphangiogenesis with resulting lymphedema in transgenic mice expressing soluble VEGF receptor-3. Nat Med 7: 199-205, 2001.
35. Hogan BM, Herpers R, Witte M, Heloterä H, Alitalo K, Duckers HJ and Schulte-Merker S: Vegfc/Flt4 signalling is suppressed by Dll4 in developing zebrafish intersegmental arteries. Development 136: 4001-4009, 2009.

36. Park JA, Kim DY, Kim YM, Lee IK and Kwon YG: Endothelial snail regulates capillary branching morphogenesis via vascular endothelial growth factor receptor 3 expression. PLoS Genet 11: e1005324, 2015.

37. Clarkin CE and Gerstenfeld LC: VEGF and bone cell signalling: An essential vessel for communication? Cell Biochem Funct 31 1-11, 2013.

38. Moslehi JJ: Cardiovascular toxic effects of targeted cancer therapies. New Engl J Med 375: 1457-1467, 2016.

(i) $(9$ This work is licensed under a Creative Commons Attribution-NonCommercial-NoDerivatives 4.0 International (CC BY-NC-ND 4.0) License. 